\title{
Monte Carlo determination of radiative properties of metal foams: comparison between idealized and real cell structures
}

\section{Salvatore Cunsolo ${ }^{1}$, Maria Oliviero ${ }^{2}$, William M. Harris ${ }^{3}$, Assunta Andreozzi ${ }^{1}$, Nicola Bianco ${ }^{*}$, Wilson K. S. Chiu ${ }^{3}$, Vincenzo Naso ${ }^{1}$}

${ }^{1}$ Dipartimento di Ingegneria Industriale - Università degli studi Federico II P.le Tecchio, 80 - 80125 - Napoli - Italy

${ }^{2}$ Istituto per i materiali compositi e biomedici Consiglio Nazionale delle Ricerche - P.le Fermi, 1 80055 Portici (Napoli) Italyt

${ }^{3}$ Department of Mechanical Engineering - University of Connecticut - Storrs - CT - U.S.A.

*Corresponding author, email: nicola.bianco@unina.it, Tel. +39 0817682645, Fax +39 0812390364 


\title{
Monte Carlo determination of radiative properties of metal foams: comparison between idealized and real cell structures
}

\author{
Salvatore Cunsolo ${ }^{1}$, Maria Oliviero ${ }^{2}$, William M. Harris ${ }^{3}$, Assunta Andreozzi ${ }^{1}$, \\ Nicola Bianco $^{1 *}$, Wilson K. S. Chiu ${ }^{3}$, Vincenzo Naso ${ }^{1}$ \\ ${ }^{1}$ Dipartimento di Ingegneria Industriale - Università degli studi Federico II \\ P.le Tecchio, 80 - 80125 - Napoli - Italy \\ ${ }^{2}$ Istituto per i materiali compositi e biomedici \\ Consiglio Nazionale delle Ricerche - P.le Fermi, 1 \\ 80055 Portici (Napoli) Italy \\ ${ }^{3}$ Department of Mechanical Engineering - University of Connecticut - Storrs - CT - U.S.A.
}

Keywords: Metallic foams, Radiative properties, X-Ray computed tomography, Monte Carlo Ray Tracing methods.

\begin{abstract}
Metal foams are being widely adopted in a number of applications relevant to heat transfer, in which many include thermal radiation as the predominant heat transfer mode. Radiative characteristics of porous media are determined either by analytical modeling or by experiments.

The aim of the present study is to evaluate the feasibility to substitute the real structure, more complex and computationally expensive, with the simpler and lighter ideal foam representation. The radiative heat transfer in foams is investigated using home-made Monte Carlo Ray Tracing codes. The geometry of the real microstructure of a metal foam is obtained by employing X-Ray computed tomography (XCT). The actual 3-D structure is imaged, from which the total porosity, surface to volume ratio and size of a representative elementary volume for thermal analysis are determined.
\end{abstract}


Results from the tomographic structures are compared to computer-generated Kelvin and WeairePhelan foam structures.

Numerical results obtained for the real and ideal geometries are compared. The agreement is good, especially when accounting for the effect of the ligament shape. The good agreement between the results obtained with reference to the Weaire-Phelan convex ligament structures and those for real structures, both in terms of extinction coefficients and scattering phase functions, suggests that such idealized structures can estimate with good accuracy the parameters for equivalent homogeneous media on the basis of the porosity and the specific surface area.

\section{Introduction}

Metal open cell foams are increasingly used in various applications where their thermal properties are of interest. Most of these applications concern relatively high temperatures and, thus, radiation propagation is an important mode of heat transfer. Therefore, many studies have already been interested in the prediction of their radiative behavior. Most of these works used analytical approaches which strongly simplify the porous architecture by considering the material as a dispersion of opaque particles of given shapes. The contributions of the various particles are usually evaluated through a combination of geometric optics approximation, diffraction theory, and Mie scattering theory and they are then summed up to evaluate the average properties of the foam under the assumption of independent scattering. Glicksman et al. [1] and Kuhn et al. [2] modeled the radiative heat transfer in cellular foam insulations by representing their structure as random arrangements of regular dodecahedron cells composed of opaque cylindrical struts and pentagonal windows. The model proposed by Doermann and Sacadura [3] improved the previous ones. The authors considered a particle modeling, obtained from the microscopic analysis of carbon open cell foams, that was more representative of the actual geometry. Kamiuto [4] studied the feasibility of using the Dul'nev's cubic unit cell model to predict analytically the radiative characteristics of an open cell foam, approximating it to a set of randomly dispersed cylinders (struts) and spheres 
(junctions). Zhao et al. [5] used a similar cubic model and an analytical view factor calculation to predict the equivalent radiative conductivity of metal foams. In order to improve the predictive capability of the Zhao's et al. model [5], Contento et al. [6] proposed a numerical approach to calculate view factors and coefficients different from those in [5]. The theoretical approach proposed by Zhao et al. [5] has been, also, used by Contento et al. [7] to develop a new radiative heat transfer model based on a more realistic representation of open cell metal foams. Loretz et al. [8] reviewed analytical models for the computation of radiative characteristics of foams for a wide variety of cells shapes and struts cross sections in order to select the model and the geometry that best simulate the radiative behavior of open cell foams. Coquard and Baillis [9] as well as Coquard et al. [10] proposed a more complex model by considering different shapes of cell (dodecahedron, cube, tetrakaidecahedron) to predict complete radiative properties (extinction, scattering and absorption coefficients, complex phase function) of low-density Expanded PolyStyrene foams (EPS). A common weak point of these analytical studies is the difficulty of accounting for the role played by the geometrical structure of the foam and the near field effects. They also typically require the knowledge of multiple geometrical parameters of the foam, such as the strut diameter or window size, which are difficult to determine univocally, because of the intrinsically random quality of the real foam structures.

Recently, the progress in 3D imaging has led to the development of numerical models which take into account much more realistic representations of the porous structure. Numerical methods can be applied both to tomographic images of real foams (real structure) and to computer generated 3D structures emulating the characteristics of real foams (ideal structure). Reference can thus be made to two approaches: the imaging-based technique and the generation-based technique.

The imaging-based technique makes use of a tomography scan of the foam, with a subsequent 3-D reconstruction of the image. In particular, after the original technique proposed by Tancrez and Taine [11], Zeghondy et al. [12, 13] and Petrasch et al. [14] applied Monte Carlo Ray Tracing (MCRT) to tomographic representations of reticulate foams in order to calculate the extinction 
coefficients and scattering phase functions. A similar technique was developed and applied by Coquard et al. [15]. The studies based on tomographic images have the advantage of accounting for the true foam structure and the near-field effects, and thus allow a substantial improvement of the predictive models for foams. However, simulations in tomographed samples require a huge computational effort and should be restricted to the study of the detailed effect of the architecture.

The generation-based technique makes use of computer-generated models of the foam. Such models can be obtained through random generation [16] or various refined periodic structures [17 19]. A common method to obtain computer generated models is to use idealized periodic structures, such as the Kelvin [20] and Phelan et al. [21] structures. Ideal structures were used by Boomsma and Poulikakos [22], Kopanidis et al. [23] and Wu et al. [24] to study convective and conjugate heat transfer at the pore level. Furthermore, the method could also be used to predict the radiative behavior of the foams.

In this study, the geometry of the real microstructure of a metal foam is obtained by employing XRay computed tomography (XCT). XCT is applied to image the actual 3-D structure, from which the total porosity, surface to volume ratio and size of a representative elementary volume for thermal analysis are determined. The Kelvin and Weaire-Phelan foam models are assumed as ideal geometries and are created using the free-to-use energy minimization software "Surface Evolver". On the basis of a Homogeneous Phase approach, equivalent homogeneous medium radiative properties are evaluated in both the real and ideal structures using home-made developed MCRT codes. Numerical results obtained for the real and ideal geometries are then compared. The final aim of the present study is to evaluate the feasibility to substitute the real structure, more complex and computationally heavier, with the simpler and lighter ideal foam representation.

\section{Foam geometry}

\subsection{Tomography based technique}

\subsubsection{Experiments}


A cylindrical foam sample (Duocel Al6101-T6, 90\% porosity, 40 PPI), $10.1 \mathrm{~mm}$ in diameter and $10.3 \mathrm{~mm}$ in height was provided by the manufacturer (Erg Aerospace Corporation, USA). The cylinder was attached to the tip of a thin wooden toothpick, of which the x-ray attenuation was negligible. The cylinder was then arranged for x-ray microtomography using an Xradia MicroXCT 400 scanner. A $1 \mathrm{X}$ objective lens was used to provide imaging capabilities with a voxel size of 44.7 $\mu \mathrm{m}$ and with the sample entirely contained within the instrument field of view. This resolution was selected in order to obtain a tomographic scan of the entire sample, still obtaining a resolution that allowed detection of the ligament structures of the foam. The x-ray projection images were reconstructed using the Xradia's filtered back-projection software [25].

The resulting 3-D data was segmented into solid and pore phases by selecting a gray level threshold using the commercial software package Avizo. Edges of the sample volume were cropped to reduce the presence of noise and imaging artifacts assignable to the tomographic reconstruction. The volume was then used in microstructural characterization codes to determine some key parameters of the foam $[26,27]$.

\subsubsection{Characteristics of the foam}

The microstructure of a typical open cell metal foam (fig.1) consists of ligaments that form a network of interconnected cells. Ligaments typically exhibits a cross sectional shape that varies from a circular to an concave triangular shape in the $0.85 \div 0.98$ foam porosity range and it is shared by three cells. Generally, the ligament geometry is characterized by the ligament length, $l$, and by the ligament diameter, $d_{l}$, often assumed as the diameter of the circle that circumscribes the cross section. The cells are irregular polyhedra. The voids inside are accessible through windows in the faces, also denoted as pores. Different definitions of the cells and pores size are found in the literature [28 - 32]. In the present study the cell diameter, $d_{c}$, is the diameter of a sphere completely

inscribed within a cell, while the pore diameter, $d_{p}$, is the diameter of the largest circle that can be inscribed within a window-like opening between neighboring cells, as sketched in fig. 1. 
In this study, the cell diameter was determined, with reference to five SEM images of the sample, by choosing five cells for each SEM image and measuring the diameter of a sphere that could be inscribed within the cells. The arithmetic mean of the diameters of the above mentioned spheres, equal to $2.64 \mathrm{~mm}$, was assumed as the cell diameter.

The pore diameter was determined making reference to cross-sectional slices taken from the reconstructed XCT image that allow an easier identification of the pores. The diameters of 31 pores were measured and their mean value was found to be equal to $0.75 \mathrm{~mm}$, that was taken as the pore diameter.

It is worth noticing that all the calculated morphological parameters are quite different from the industrial specifications. This disagreement was also found by Petrasch et. al. [32] and Howell et al. [33].

The porosity of the foam, $\varepsilon$, was determined by voxel-based counting schemes and was found to be equal to $89.4 \%$. The specific surface area, $S / V$, was measured in two different ways: either by creating a triangular mesh of the surface of the structure and then evaluating the surface of the triangles or by means of a 2-point correlation function directly calculated using the segmented data, following the method outlined in [32]. These methods yielded a 1,064 1/m and 1,037 1/m specific surface area, respectively, averaging $S / V=1,0501 / \mathrm{m}$.

\subsubsection{Representative Volume Element (RVE)}

Selection of a Representative Volume Element (RVE) from the 3-D data set provides a simulation domain and enables the study of transport phenomena at the pore scale. To ensure that the extracted RVE has the same radiative behavior as the overall foam structure, values of specific surface area and porosity have to be as similar as possible.

A statistical RVE (SRVE) was used to check that the above-mentioned properties are independent of the RVE position and size, in agreement with Zhang et al. [34]. The SRVE is defined as the representative volume element below which the mean and standard deviation of the quantity of 
interest vary significantly with the RVE size. In this study, cubic RVEs extracted from the central region of the digital foam structure have been considered. The porosity and specific surface area, descriptors of the foam structure, are evaluated for a range of different RVE volume sizes, obtained varying the edge length, $L_{e d g}$. For each $L_{e d g}$. several RVEs are extracted and the mean as well as the standard deviation of the above said descriptors are evaluated. Figure 2 shows the mean values of the porosity and the specific surface area, with the associated errors, as a function of the normalized edge length of RVE, $z^{*}=L_{e d g} / d_{c}$. Dashed lines represent the error curves, obtained by adding or subtracting the standard deviation to the mean value. A coefficient of variation, $\mathrm{CV}$, defined as the standard deviation normalized by the mean, has been introduced. RVEs that can be chosen are those that have a normalized edge length characterized by a $z^{*}$ value larger than the $z^{*}$ obtained with a $5 \% \mathrm{CV}$ value (see shaded area), which is seen to occur at $z^{*}=0.6$ and $z^{*}=1.8$ for the porosity and specific surface area, respectively. To be conservative, a $z^{*}$ value of 2 is used in the following,

yielding a RVE with dimensions $5.28 \times 5.28 \times 5.28 \mathrm{~mm}^{3}$. The significant geometrical parameters of the domain are: RVE sizes $=5.28 \times 5.28 \times 5.28 \mathrm{~mm}^{3} ; \varepsilon=88.96 \% ; S / V=1,0561 / \mathrm{m}$.

\subsection{Generation based technique and ligament shape correction}

When using computer-generated structures, the foam structure is approximated by a periodically repeated base unit (cell), that turns out to be the RVE. Two different ideal representations are used: that proposed by Lord Kelvin [20] and that proposed by Phelan et al. [21]. The unit cell proposed by Kelvin [20] is a tetrakaidecahedron with slightly curved faces. The unit cell proposed by Phelan et al. [21] is made up, instead, of eight equal-volume polyhedral: six 14-sided cells (with 12 pentagonal, and 2 hexagonal faces) and two pentagonal dodecahedra. The latter representation is usually considered an improvement over the former: its surface energy is smaller [21] and it exhibits the prevailing number of pentagonal faces commonly observed in real foams.

Both geometries are defined in an input file in the "Surface Evolver" surface energy minimization software, as described by Brakke [35]. After a first energy minimization process, the resulting 
structure is "wet" (i.e. the solid is added along the borders of the foam, the so called "Plateau borders"). Then the porosity is adjusted to the desired level, in order to match the porosity of the real, imaged structure. A further energy minimization is accomplished until a stable structure is obtained. The resulting structure can be readily exported in a triangular mesh format, which, after being scaled to match the $S / V$ ratios of the imaged structure, can be directly used in the Monte Carlo codes.

While building the numerical structures, it was observed that the structures tend to present a very marked concave shape of the ligaments, while the real foam tends to exhibit a convex shape, as shown in fig. 3a - c. This is probably because by default the Surface Evolver software generates structures based only on the principle of surface energy minimization, i.e. surface tension dominated structures, but in reality during the process of foam formation other forces, such as gravity and diffusion forces, play a significant role, especially at relatively lower porosities. To compare the effect of the ligament shape on the results, a second set of ideal structures was generated, this time adjusting the Surface Evolver generation parameters in such a way as to obtain convex shapes for the ligaments. An overview of the typical foam structures used in this study is reported in fig. $4 \mathrm{a}-\mathrm{c}$, where grey scale is used to improve the spatial resolution of the image.

\section{Methodology}

The Homogeneous Phase Approach (HPA) is used in this work. It implies that the radiative behavior of a composite medium can be matched faithfully by an equivalent homogeneous semitransparent medium. Therefore, the radiation propagation is described using a unique homogenized radiation intensity and the radiative behavior of the foams is described by spectral radiative characteristics (extinction coefficient, $\beta_{\lambda}$, absorption coefficient, $\kappa_{\lambda}$, scattering coefficient, $\sigma_{\lambda}$, scattering phase function, $\left.\Phi_{\lambda}(\mu)\right)$, refractive index $n$ ). Considering that the propagation of radiation only occurs in the transparent phase, the refractive index is generally assumed to be equal to that of the transparent phase. On the other hand, a method to calculate the values of the other above- 
mentioned properties is to be chosen, such that an equivalent homogeneous medium may be defined. The Monte Carlo methods developed in [14] and [15] have been adapted and applied to both tomographic images and computer-generated structures in this work.

The study has been simplified by making the assumptions reported in the following.

Geometric optics has been assumed to be valid, and diffraction has been neglected. This assumption can be considered acceptable for the investigated foam at wavelengths shorter than $\lambda=60 \mu \mathrm{m}$. In fact the smallest feature size of the foam is the ligament diameter $\left(d_{l} \approx 100 \mu \mathrm{m}\right)$, so in the aforementioned wavelength range the size parameter, $2 \pi d_{l} / \lambda$, is larger than 10 , which is the generally accepted limit of validity for geometric optics [36]. It is worth noticing that this wavelength range covers more than $95 \%$ of the energy emitted by a blackbody at room temperature, so the approximation does not substantially limit the applicability of the model at temperatures higher than the ambient.

Statistical homogeneity of the medium has to be assumed to make the HPA formulation possible, i.e. the medium must be homogeneous at least at some statistical level to allow to model it as an equivalent continuum. Additionally, isotropy is assumed, any anisotropies in the $x, y, z$ directions in the structures being neglected, by summing up together all contributions from different directions without distinction.

Considering the struts opaque, which is very acceptable for most metals, allows us to neglect the propagation of radiation in the solid part.

Though the analysis could have been carried out with reference to spectral quantities, for the sake of brevity, solids have been assumed to be grey, thus allowing to consider all properties independent of the wavelength.

The reflectivity has been assumed to be independent of the incidence angle. This is a good assumption for diffuse reflection, and has also been used for specular reflection $[14,15]$. It considerably simplifies the evaluation of scattering and absorption coefficients, as it will be shown in the following. 
Finally, the fluid has been assumed to be transparent, so that no extinction of the radiation intensity occurs in it; as a consequence, only the solid/fluid interface actually participates to radiation heat transfer.

\subsection{Monte Carlo method}

A large number of stochastic rays are cast and their histories are tracked until they intersect the solid/fluid interface. Details about ray generation process are given in subsection 3.1.2. The distance traveled by each ray before the intersection is stored. Then a pair of reflected rays (one specular and one diffuse) are generated and the angle between the incident ray and the reflected rays is calculated and stored.

According to [19], a cumulative free path distribution function, $G(s)$ is then calculated as

$$
G(s)=\int_{0}^{s} \frac{1}{N} \sum_{n=1}^{N} \delta\left(s^{\prime}-s_{n}\right) \mathrm{d} s^{\prime}
$$

where $\delta$ is the Dirac delta function, $N$ is the total number of rays cast, $s$ is the path length and $s_{n}$ is the path length of the $n$-th realization.

The correlation between the $G(s)$ function and the normalized radiation intensity over a path, $I_{n}(s)=$ $I(s) / I_{0}$, with $I_{0}$ the incident radiation intensity, is

$$
I_{n}(s) \approx 1-G(s)
$$

that is then related to the extinction coefficient, $\beta$, through the Beer-Lambert-Bouguer law

$$
I_{n}(s) \approx \mathrm{e}^{-\beta s}
$$

where $\beta$ is found through a least squares fitting. The coefficient of determination is high in most practical cases; in the present study $R^{2}$ higher than 0.97 have been found in all cases. Finally, accounting for the angular independence of the reflectivity, the absorption coefficient, $\kappa$, and the scattering coefficient, $\sigma$, are calculated as $k=\alpha \beta$ and $\sigma=\rho \beta$, where $\alpha$ and $\rho$ are the absorptivity and 
reflectivity of the solid surface, respectively. The values of $\alpha$ and $\rho$ are known for many materials and can be found using various techniques [36].

Following the approach outlined in $[19,20]$, the scattering phase function, $\Phi(\mu)$, can be calculated as

$$
\phi(\mu)=\frac{1}{N} \sum_{n=1}^{N} \delta\left(\mu-\mu_{n}\right)
$$

with $\mu$ the scattering angle cosine and $\mu_{n}$ the scattering angle cosine of the $n$th realization.

Subtle but potentially significant differences, especially regarding the ray casting methods and the mathematical representation of the foam, exist between various authors and will be discussed in the following.

\subsubsection{Mathematical representation of the foam}

As it was above mentioned, there are two ways to represent the foam in the 3-D space:

- A polygon mesh, where the interface is represented by a set of 3-D triangles. The stochastic rays are tested for intersection against the triangles using simple geometrical rules. The 3-D mesh is obtained from the volumetric data, using the free software iso2mesh [37].

- A continuous 3-D density function, generated from the voxel representation of the foam, using a linear shape function. The voxels are considered to be the vertices of a cubic lattice and the value of the function at each point is given by the trilinear interpolation of the values of vertices of the cube to which the point belongs. The interface is, therefore, represented as an isosurface of this function. The stochastic rays are tested for intersection against the interface by iteratively checking the value of the density function along the ray.

The sensitivity of the results to the representation is tested in terms of the free path distribution function in the tomographic structure.

The normalized radiation intensity, linked to the free path distribution function by Eq. 2, is presented in fig. 5 as a function of the path length for the two different representations. Very small 
differences $(<5 \%)$ are exhibited and can be ascribed to the differences between the shape functions used in iso2mesh and the shape functions used in the density function representation.

In the following, in order to minimize the number of data format transformations, each dataset has been processed in its "native", format, i.e. a continuous density function has been used to represent tomographic structures, while polygonal meshes have been used for generated structures.

\subsubsection{Ray generation process}

Stochastic rays can be cast either from random points uniformly distributed within the void region, as in [14], or from random points uniformly distributed over the foam surface, as in [15].

The sensitivity of the results to the representation is again tested in terms of the free path distribution function in the tomographic structure. The normalized radiation intensity as a function of the path length for the two different ray casting techniques is presented in fig.6. One can notice that the differences between the $\beta$ values are larger than 25\%, and, therefore, they are significant. The largest differences are exhibited in the near-field region. This was to be expected because of the geometry of the foam, consisting of void cells enclosed by solid ligaments, that orients toward the void region rays cast from the solid ligaments.

In the following, rays are cast from the random points uniformly distributed within the void region. This approach was chosen since casting rays from the entire foam region is thought to be closer to the actual physical phenomenon under modeling. In fact, if the foam were directly irradiated by an external source, for example in a photometric experiment, the radiation would come from all the "void region" rather than only from the solid/void interface. Thus results obtained in this way should be more easily comparable with experimental data and applicable to modeling a real apparatus.

\subsubsection{Monte Carlo convergence}


To check the numerical convergence of the Monte Carlo algorithm, computations were run with $N$ $=10^{4}, 10^{5}, 10^{6}$, where $N$ denotes the number of rays. The calculated values of the average extinction coefficient, as a function of the number of rays cast, together with the $95 \%$ confidence interval is presented in fig.7. An $N=10^{6}$ number of rays with a relative error equal to $0.25 \%$ is used in following computations.

In order to check the sampling convergence, the original voxel structure was down-sampled by factors 1.5, 2.0, 3.0, 4.0, and for each down-sampled structure a Monte Carlo computation was run. The down-sampling procedure works as follows. For a down-sampling factor $\mathrm{M}$, from an AxBxC grid a new $(\mathrm{A} / \mathrm{M}) \mathrm{x}(\mathrm{B} / \mathrm{M}) \mathrm{x}(\mathrm{C} / \mathrm{M})$ grid is created; the value of each new element is equal to the rounded average of the corresponding $\mathrm{MxMxM}$ elements on the original grid. The process is extended to a rational $\mathrm{M} / \mathrm{N}$ down-sampling factor by applying an $\mathrm{N}$ factor scaling to the original grid.

The extinction coefficient as a function of the down-sampling factor is reported in fig.8, which exhibits the convergence of the down-sampled results to the "full sampled" result. The figure shows that down-sampling by factors 2 and 1.5 gives values within $\pm 2 \%$ from the full sampled original grid (corresponding to the "1" down-sampling); one can, therefore, conclude that sampling effects are negligible.

\subsubsection{Monte Carlo validation}

In order to validate the code, three sets of Identical Overlapping Opaque solid Spheres (IOOS) in a transparent fluid were generated, with specific surface area ratios ranging from $1001 / \mathrm{m}$ to $4001 / \mathrm{m}$ and porosities ranging from $87 \%$ to $99 \%$. The above mentioned ranges were chosen such as to span from the optically thin limit to values typical of foams. The extinction coefficient was calculated and presented in fig.9, together with the $\beta=(S / V) / 4$ analytical relationship given by Tancrez and Taine [11]. The figure exhibits a very good agreement between $\beta$ values obtained by simulation and those obtained analytically. 
A set of Identical Overlapping Transparent fluid Spheres (IOTS) in a solid phase was also generated. The incident angle cosine distributions for both IOOS and IOTS sets were calculated and are reported in fig 10, together with some points taken from the distributions presented by Petrasch et al. [14]. It is worth remarking that this is not the distribution of scattering angles, i.e. relative angles between incident and scattered rays, but, rather, of incident angles, i.e. angles between incident rays and surface normal. Also in this case results are in very good agreement.

\section{Results and discussion}

The free path distribution functions, the consequent extinction coefficients and the scattering phase functions have been estimated both for the real and idealized structures. For the sake of clarity, two comparisons have been reported for each property: the former compares the real structure (Tomo) and the Kelvin (K) and Weaire-Phelan (WP) idealized structures without any ligament shape correction (i.e. with concave ligaments); the latter compares the real structure and the idealized structures with ligament shape correction (i.e. with convex ligaments).

\subsection{Normalized radiation intensity and extinction coefficient}

The normalized radiation intensity as a function of the path length, for the real structure (Tomo) and the Kelvin (K) and Weaire-Phelan (WP) idealized structures, both without and with ligament shape correction, are presented in fig. 11a and fig.11b, respectively.

The comparison between the real and the ideal structures clearly shows that the effects of the ligament shape are very marked, the difference between the calculated values of $\beta$ for the concave and the convex ligament ideal structures being larger than $10 \%$. This effect can be attributed to the fact that the distance between the center and the extremities of the ligament in its cross section is larger in a concave ligament than in a convex ligament, the area of the section being the same (see

fig.3). Therefore, concave ligaments obstruct a larger fraction of the pore windows for a given specific surface area, thus generating a stronger extinction in the porous media as a whole. It is also 
worth noticing that ideal structures with convex ligaments provide results closer to those of the real structure: switching from concave to convex ligaments, the difference between results for the real and the ideal structures drops from $\sim 20 \%$ to $\sim 10 \%$.

It can also be noticed that the Weaire-Phelan structure results agree with the tomographic structure marginally better than the Kelvin structures, with a nearly 3\% difference. The most likely explanation for this is that, though marginally, Weaire-Phelan structures are a geometric reproduction of the real foam structure more accurate than the Kelvin structures.

\subsection{Scattering phase function}

The scattering phase functions are calculated by evaluating the distribution of the angles between incident rays and reflected rays, which strongly depends on the kind of reflection assumed. While Monte Carlo simulation does virtually support a wide range of possible bidirectional reflectance distribution functions (BDRF), this works mainly focuses on geometry, rather than surface characteristics. Therefore, for the sake of simplicity only purely specular reflection and purely diffuse reflection, with reflectance independent scattering angle, are considered in this work. In the former case, the scattering angle is twice the angle between the incident angle and the local surface normal, while in the latter case, after identifying the surface normal, a random ray is cast extracting its direction randomly from the diffuse surface emission distribution and the scattering angle is given by the angle between the incident ray and this newly generated random ray.

The scattering phase function as a function of the scattering angle cosine, for purely specular and diffuse reflections, are presented in fig.12a and fig.12b for concave and convex ligaments, respectively. It can be remarked that the shape of both phase functions match quite closely those presented in [19]. One can also notice that the ideal structures match the real structure quite well, especially when reference is made to the diffuse reflection.

It is also worth pointing out that, as it was remarked with reference to the normalized radiation intensity, the results for the convex ligament structures and for the Weaire-Phelan structure match 
the results for the tomographic structure better than the results for the concave ligament structures and Kelvin structures. In order to quantify the fit between each of the scattering phase functions obtained from the ideal structures and the corresponding scattering phase functions from the tomographic structure, the coefficient of determination $R^{2}$ was calculated as:

$$
R^{2}=1-\frac{\int_{-1}^{1}\left[\Phi_{\text {id }}(\mu)-\Phi_{\text {Toтo }}(\mu)\right]^{2} \mathrm{~d} \mu}{\int_{-1}^{1}\left[\Phi_{\text {Tomo }}(\mu)-\overline{\left.\Phi_{\text {Tomo }}(\mu)\right]^{2} \mathrm{~d} \mu}\right.}
$$

where the bar denotes an average value.

The values of the coefficient of determination for the specular scattering phase function are: 0.47 for the Kelvin concave ligament; 0.65 for the Weaire-Phelan concave ligament; 0.73 for the Kelvin convex ligament; 0.87 for the Weaire-Phelan convex ligament.

As it was already remarked with reference to the extinction coefficient, also for scattering function the convex ligament structures provide better agreement than the concave ligament structures, and the Weaire-Phelan structures provide better agreement than the Kelvin structures. This seems to confirm that the choice of the more faithful geometry does indeed increase overall agreement between predictions of generated and real geometries.

\section{Conclusions}

In this study, radiation heat transfer inside reticulated foams was investigated through the development of Monte Carlo Ray Tracing algorithms.

A set of properties was evaluated that characterize a homogeneous medium whose behavior most closely approximates that of the reticulated foam structure. The key features and critical points of the method were highlighted and a comparison was carried out to ascertain differences between the approaches surveyed from literature. In particular, the comparison pointed out that the choice of the

ray casting method strongly affects the estimate of parameters for the equivalent homogeneous medium. 
The algorithms were then applied to tomographic structures as well as computer-generated Kelvin and Weaire-Phelan foam structures.

The sensitivity of the results to microstructural parameters of the idealized structures was evaluated, with particular reference to the ligament shape and the generating cell arrangement.

The good agreement between the results obtained with reference to the Weaire-Phelan convex ligament structures and those for real structures, both for extinction coefficients and scattering phase functions, suggests that such idealized structures can estimate with good accuracy the parameters for equivalent homogeneous media on the basis of the porosity and the specific surface area.

The use of ideal structures with convex ligaments (more closely matching the real structure) was found to match the results for the real structure more accurately than those for the ideal concave ligament structures.

It was also found that the Weaire-Phelan structure, in comparison with the Kelvin structure, provides results that are in marginally better agreement with those obtained for the real foam. The most likely explanation for this is that, though marginally, Weaire-Phelan structures are a geometric reproduction of the real foam structure more accurate than the Kelvin structures.

\section{Nomenclature}

\section{Latin symbols}

d Diameter (m)

$G(s) \quad$ Cumulative free path distribution function

I Radiation intensity $\left(\mathrm{W} / \mathrm{m}^{2} \mathrm{sr}\right)$

$l \quad$ Ligament length (m)

$L_{\text {edg }} \quad$ Edge length (m)

n Refractive index

$N \quad$ Total number of rays cast 
PPI Pores per inch (1/in)

$R^{2} \quad$ Coefficient of determination

$s \quad$ Path length (m)

$s_{n} \quad$ Path length of the n-th realization (m)

$S / V \quad$ Specific surface area $(1 / \mathrm{m})$

$z^{*} \quad$ Normalized edge length

\section{Greek symbols}

$\alpha \quad$ Solid surface absorptivity

$\beta \quad$ Extinction coefficient $(1 / \mathrm{m})$

$\delta \quad$ Dirac delta function

$\varepsilon \quad$ Porosity

$\kappa \quad$ Absorption coefficient (1/m)

$\lambda \quad$ Wavelength (m)

$\sigma \quad$ Scattering coefficient $(1 / \mathrm{m})$

$\mu \quad$ Scattering angle cosine

$\mu_{n} \quad$ Scattering angle cosine of the $\mathrm{n}$-th realization

$\Phi(\mu) \quad$ Scattering phase function

$\rho \quad$ Solid surface reflectivity

\section{Subscripts}

$0 \quad$ Incident radiation

c Cell

$l \quad$ Ligament

n Normalized radiation

$p \quad$ Pore

id Ideal

$\lambda \quad$ Spectral 


\section{Tomo Tomographic}

\section{Acknowledgements}

This work was carried out within an Agreement between the University of Connecticut and the Università di Napoli Federico II and was supported by Italian Government MIUR grant PRIN2009KSSKL3. W.M. Harris and W.K.S. Chiu acknowledge financial support from an Energy Frontier Research Center on Science Based Nano-Structure Design and Synthesis of Heterogeneous Functional Materials for Energy Systems (HeteroFoaM Center) funded by the U.S. Department of Energy, Office of Science, Office of Basic Energy Sciences (Award DE-SC0001061). The authors acknowledge Sina Shahbazmohamadi for his help in x-ray microtomography measurements at the University of Connecticut's Center for Clean Energy Engineering.

\section{References}

[1] L.R. Glicksmann, M.A. Schuetz, M. Sinofsky, Radiation heat transfer in foam insulation, J. Heat Transfer 109 (1987) 809-812.

[2] J. Kuhn, H.P. Ebert, M.C. Arduini Schuster, D. Buttner, J. Fricke, Thermal transport in polystyrene and polyurethanes foam insulations, Int. J. Heat Mass Transfer 35 (1992), 1795-1801.

[3] D. Doermann, J.F. Sacadura, Heat Transfer in open cell foam insulation, J. Heat Transfer 118 (1996) 88-93.

[4] K. Kamiuto, Study of Dul'nev's model for the thermal and radiative properties of open-cellular porous material, JSME Int. J. Series B 4 (1997) 577-582.

[5] C.Y. Zhao, S.A. Tassou, T.J. Lu, Analytical considerations of thermal radiation in cellular metal foams with open cells, Int. J. Heat Mass Transfer 51 (2008) 929-940.

[6] G. Contento, M. Oliviero, N. Bianco, V. Naso, Prediction of radiative heat transfer in metallic foams, Int. J. Thermal Sciences 76 (2014) 147-154. 
[7] G. Contento, M. Oliviero, N. Bianco, V. Naso, The prediction of radiation heat transfer in open cell metal foams by a model based on the Lord Kelvin representation, Int. J. Heat Mass Transfer 76 (2014) 499-508.

[8] M. Loretz, R. Coquard, D. Baillis, E. Maire, Metallic foams: radiative properties/comparison between different models, J. Quant. Spectrosc. Radiat. Transfer 109 (2008) 16-27.

[9] R. Coquard, D. Baillis, Modelling of heat transfer in low-density EPS foams, J. Heat Transfer 128 (2006) 538-549.

[10] R. Coquard, D. Baillis, D. Quenard, Radiative properties of expanded polystyrene foams, J. Heat Transfer 131 (2009) 012702.1- 012702.10.

[11] M. Tancrez, J. Taine, Direct identification of absorption and scattering coefficients and phase function of a porous medium by a Monte Carlo technique, Int. J. Heat Mass Transfer 47 (2004) 373383.

[12] B. Zeghondy, E. Iacona, J. Taine, Determination of the anisotropic radiative properties of a porous material by radiative distribution function identification (RDFI), Int. J. Heat Mass Transfer 49 (2006) 2810-2119.

[13] B. Zeghondy, E. Iacona, J. Taine, Experimental and RDFI calculated radiative properties of a mullite foam, Int. J. Heat Mass Transfer 49 (2006) 3702-3707.

[14] J. Petrasch, P. Wyss, A. Steinfeld, Tomography-based Monte Carlo determination of radiative properties of reticulate porous ceramics, J. Quantitative Spectroscopy Radiative Transfer 105 (2007) 180-197.

[15] R. Coquard, D. Baillis, J. Randrianalisoa, Homogeneous phase and multi-phase approaches for modeling radiative transfer in foams, Int. J. Thermal Sciences 50 (2011) 1648-1663.

[16] M. Wang, N. Pan, Modeling and prediction of the effective thermal conductivity of random open-cell porous foams , Int. J. Heat Mass Transfer 51 (2008) 1325-1331.

[17] F. Kuwahara, T. Yamane, A. Nakayama, Large eddy simulation of turbulent flow in porous media, Int. Comm. Heat Mass Transfer 33 (2006) 411-418. 
[18] F. Kuwahara, M. Shirota, A. Nakayama, A numerical study of interfacial convective heat transfer coefficient in two-energy equation model for convection in porous media, Int. J. Heat Mass Transfer 44 (2001) 1153-1159.

[19] I. Ghosh, Heat-transfer analysis of high porosity open-cell metal foam, J. Heat Transfer 130 (2008) 034501-034506.

[20] Lord Kelvin (Sir W. Thomson), On the division of space with minimum partitional area, Acta Mathematica 11 (1887) 121-134.

[21] R. Phelan, D. Weaire, K. Brakke, Computation of equilibrium foam structures using the Surface Evolver Experimental Mathematics 4 (1995) 181-191.

[22] K. Boomsma, D. Poulikakos, On the effective thermal conductivity of a three dimensionally structured fluid-saturated metal foam, Int. J. Heat Fluid Flow 44 (2001) 825-834.

[23] A. Kopanidis, A. Theodorakakos, E. Gavaises, D. Bouris, 3D numerical simulation of flow and conjugate heat transfer through a porescale model of high porosity open cell metal foam, Int. J. Heat Mass Transfer 53 (2010) 2539-2550.

[24] Z. Wu, C. Caliot, G. Flamant, Z. Wang, Numerical simulation of convective heat transfer between air flow and ceramic foams to optimise volumetric solar air receiver performances, Int. J. Heat Mass Transfer 54 (2011) 1527-37.

[25] J.R. Izzo, A.S. Joshi, A.A. Peracchio, K.N. Grew, W.K. S. Chiu, A.T. Tkachuk, S.H. Wang, W. Yun, Non-destructive reconstruction and analysis of solid oxide fuel cell anodes using x-ray computed tomography at Sub-50 nm resolution, J. Electrochemical Society 155 (2008) 504-508.

[26] K.N. Grew, A.A. Peracchio, A.S. Joshi, J.R. Izzo, W.K.S Chiu, Characterization and analysis methods for the examination of the heterogeneous solid oxide fuel cell electrode microstructure. Part 1: Volumetric measurements of the heterogeneous structure, J. Power Sources 195 (2010) 7930-7942.

[27] K.N. Grew, A.A. Peracchio, W.K.S Chiu, Characterization and analysis methods for the examination of the heterogeneous solid oxide fuel cell electrode microstructure: Part 2. Quantitative 
measurement of the microstructure and contributions to transport losses, J. Power Sources 195 (2010) 7943-7958.

[28] L. J. Gibson, M. F. Ashby, Cellular solids: Structure and properties, Cambridge University Press, 1999.

[29] S. Mahjoob, K. Vafai, A synthesis of fluid and thermal transport models for metal foam heat exchangers, Int. J. Heat Mass Transfer 51 (2008) 3701-3711.

[30] A. J. Fuller, T. Kim, H. P. Hodson, T. J. Lu, Measurement and interpretation of the heat transfer coefficients of metal foams, Proceedings of the Institution of Mechanical Engineers, Part C: Journal of Mechanical Engineering Science 219 (2005), 183-191.

[31] E. Maire, P. Colombo, J. Adrien, L. Babout, L. Biasetto, Characterization of the morphology of cellular ceramics by 3D image processing of X-ray tomography, J. European Ceramic Society, 27(2007), 1973-1981.

[32] J. Petrasch, F. Meier, H. Friess, A. Steinfeld, Tomography based determination of permeability, Dupuit-Forchheimer coefficient, and interfacial heat transfer coefficient in reticulate porous ceramics, Int. J. Heat Fluid Flow 29 (2008) 315-326.

[33] J. Howell, M. Hall, J. Ellzey, Combustion of hydrocarbon fuels within porous inert media, Progress in Energy Combustion Science 22 (1999) 121-145.

[34] D. Zhang, R. Zhang, S. Chen, W.E Soll, Pore scale study of flow in porous media: Scale dependency, REV, and statistical REV, Geophysical Research Letters 27 (2000) 1195-1198.

[35] K.A. Brakke, The surface evolver, Experimental Mathematics 1 (1992) 141-165.

[36] R. Siegel, Thermal radiation heat transfer, Taylor \& Francis (2001).

[37] http://iso2mesh.sourceforge.net (last accessed 19 November 2013). 


\section{Captions to the figures}

Figure 1. SEM image of the sample: pore diameter, $d_{p}$, and cell diameter, $d_{c}$.

Figure 2. Mean porosity and mean specific surface area $v s$ normalized square section side of the RVE (solid lines).

Figure 3. Typical ligament cross sections: (a) Tomographic structure; (b) Concave ideal structures; (c) Convex ideal structures.

Figure 4. Examples of foam structures: (a) Tomographic structure; (b) Concave Kelvin structure; (c) Convex Weaire-Phelan structure.

Figure 5. Normalized radiation intensity over a path $v s$. path length: two different representations.

Figure 6. Normalized radiation intensity over a path $v s$. path length: two different ray casting techniques.

Figure 7. Average extinction coefficient and $95 \%$ confidence interval $v s$. number of rays cast.

Figure 8. Extinction coefficient $v s$. downsampling factor.

Figure 9. Extinction coefficient $v s$. specific surface area in IOOS sets: analytical relationship and simulation.

Figure 10. Incident angle cosine distribution $v s$. incident angle cosine: IOOS and IOTS sets.

Figure 11. Normalized radiation intensity vs. path length, for the real and ideal structures: a) concave ligaments; b) convex ligaments.

Figure 12. Scattering phase function $v s$. scattering angle cosine, for the real and the ideal structures: a) concave ligaments; b) convex ligaments. 


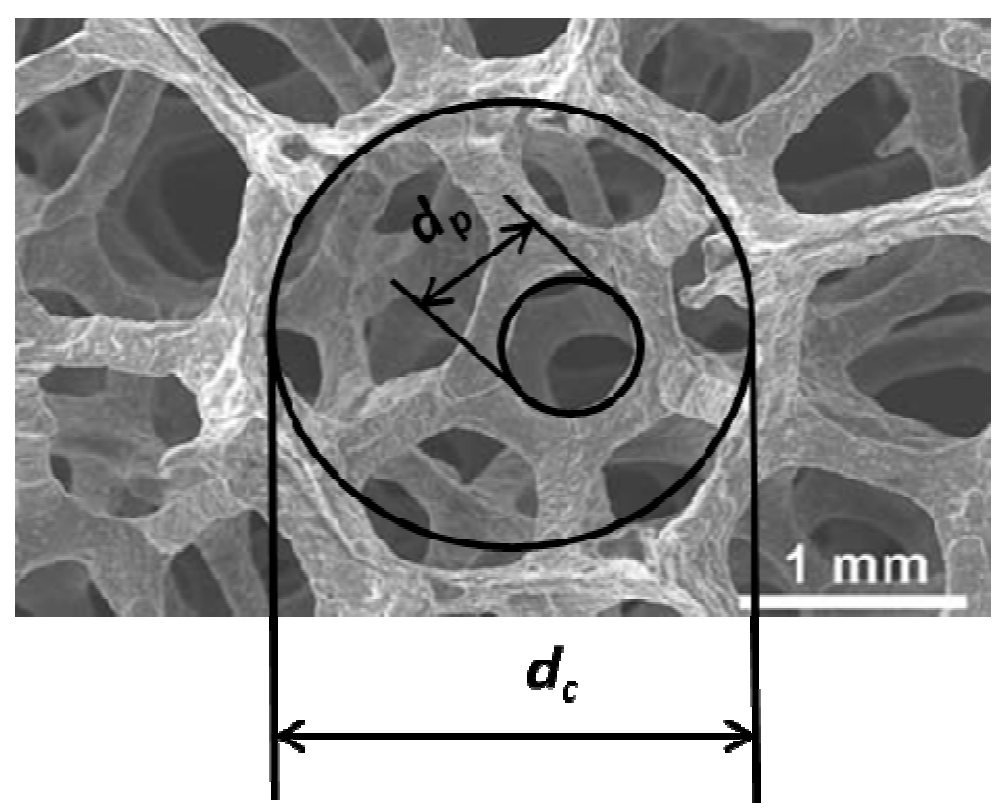

Figure 1 

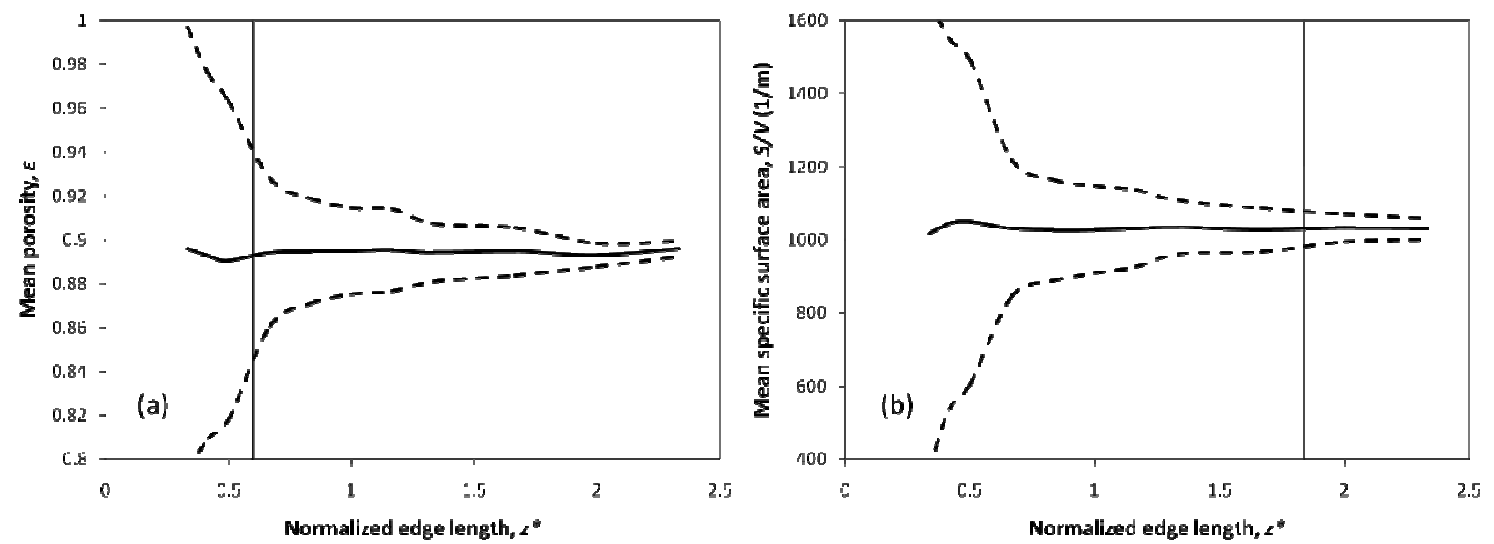

Figure 2 

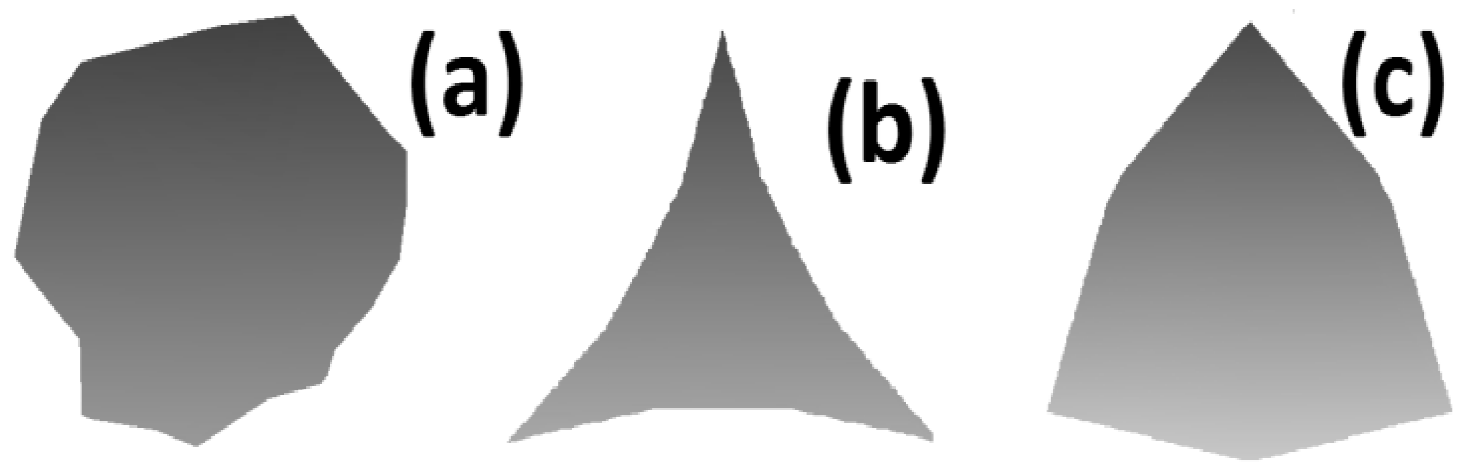

Figure 3 

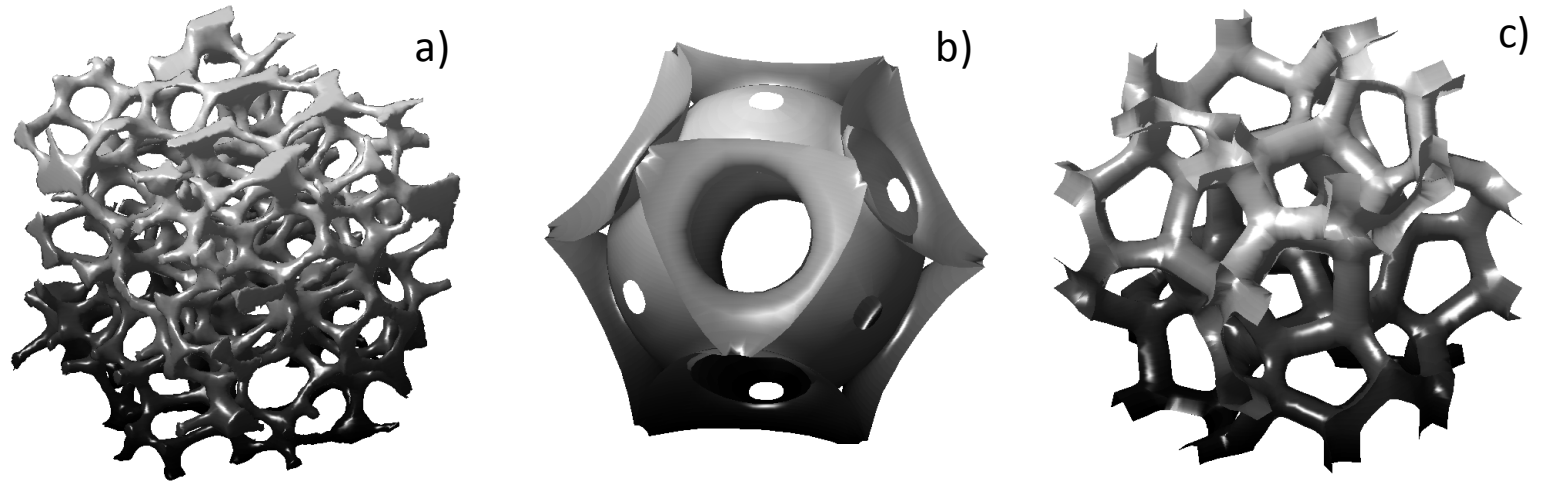

Figure 4 


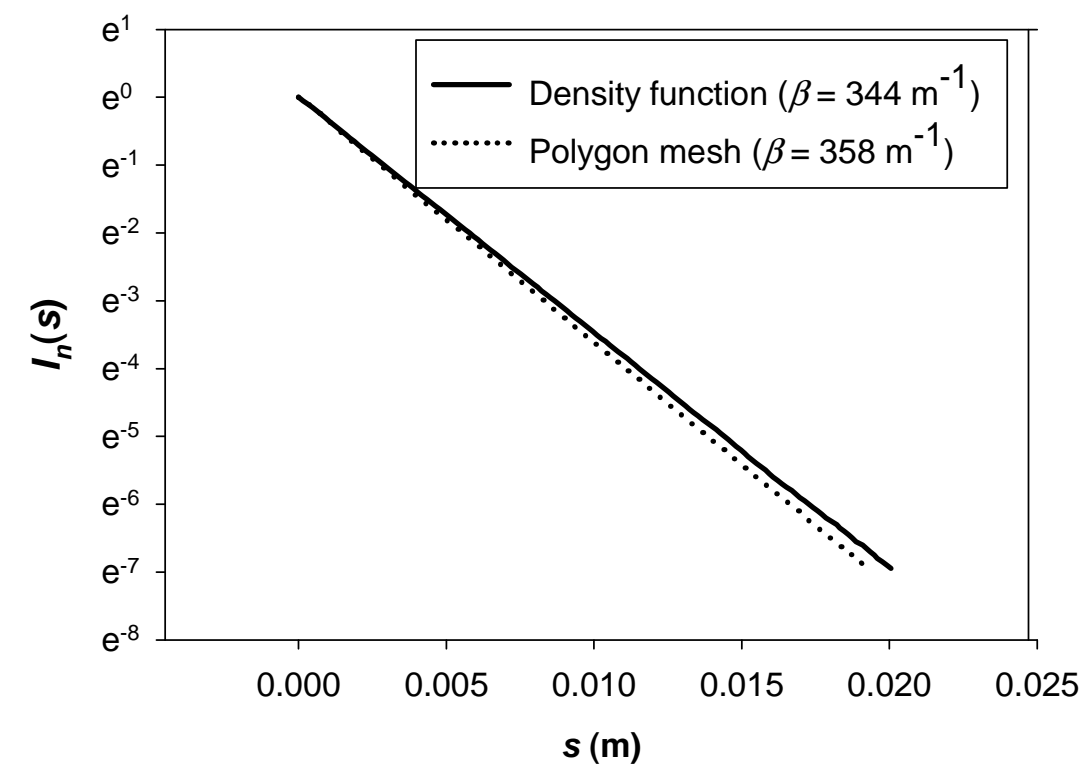

Figure 5 


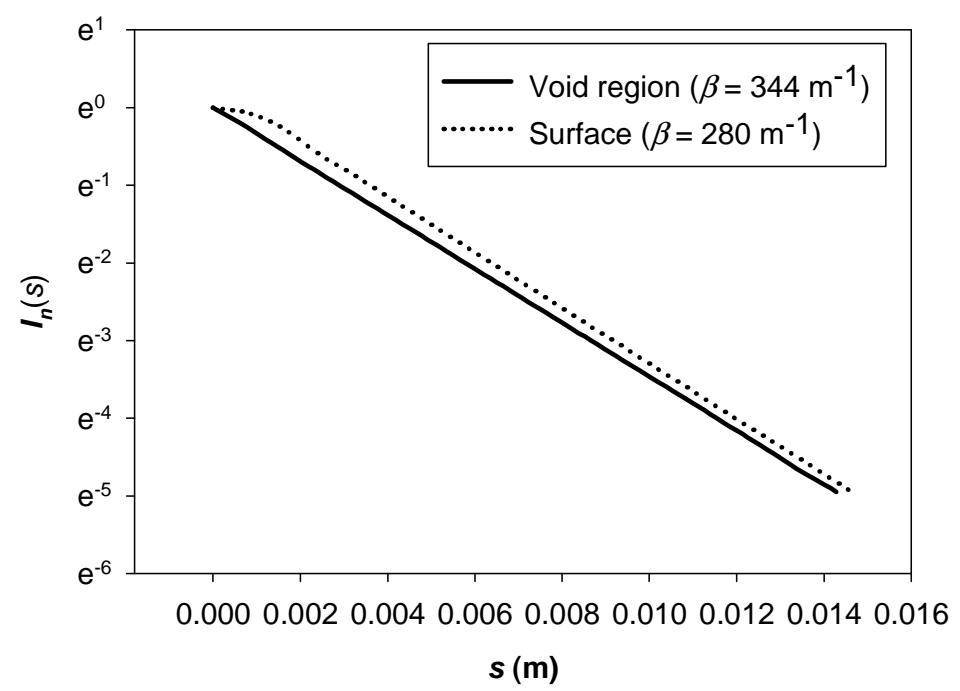

Figure 6 


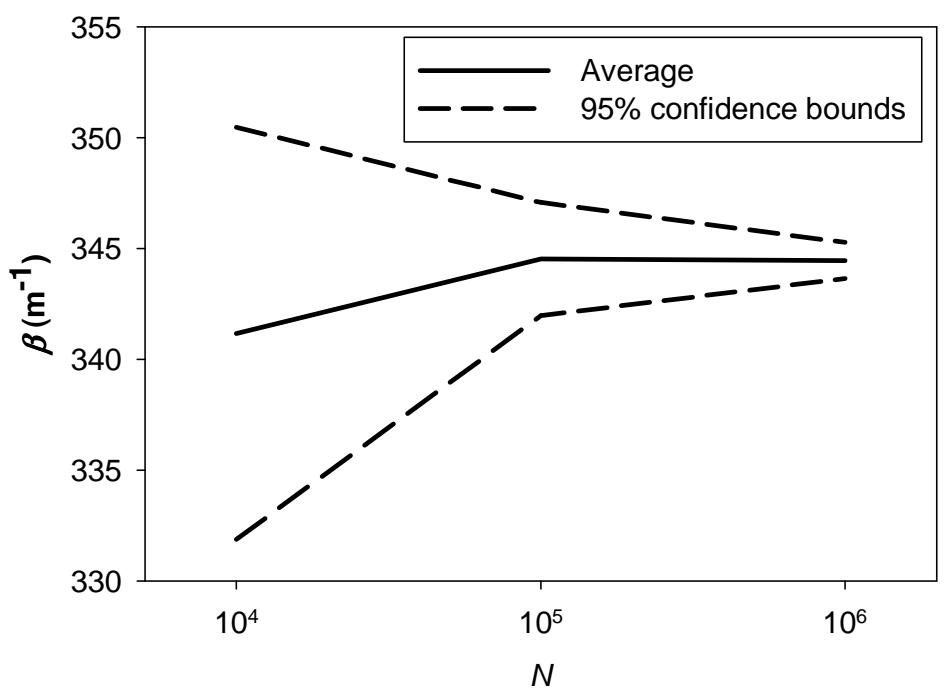

Figure 7 


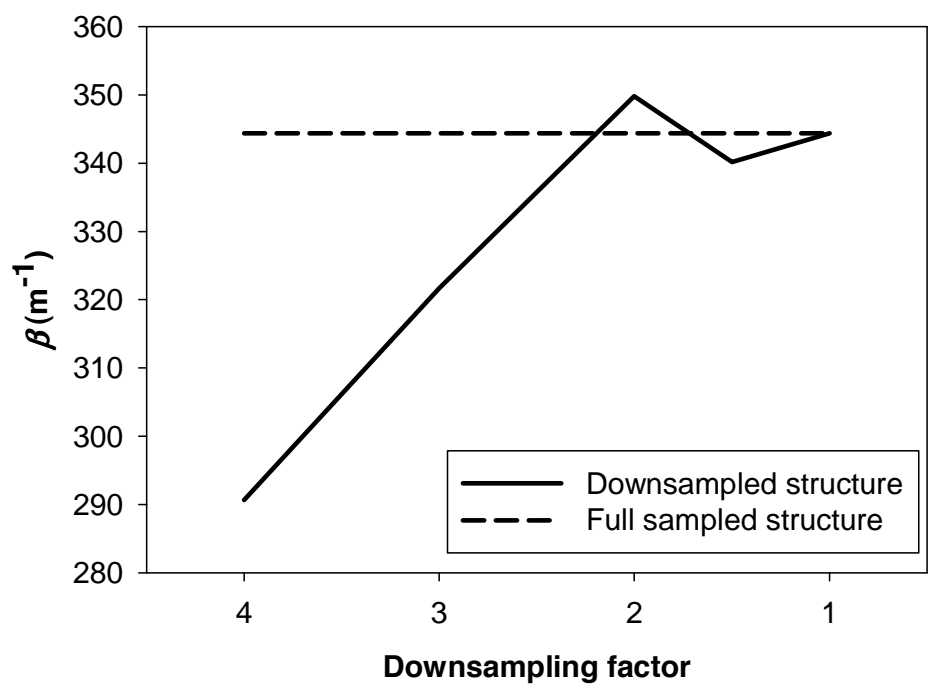

Figure 8 


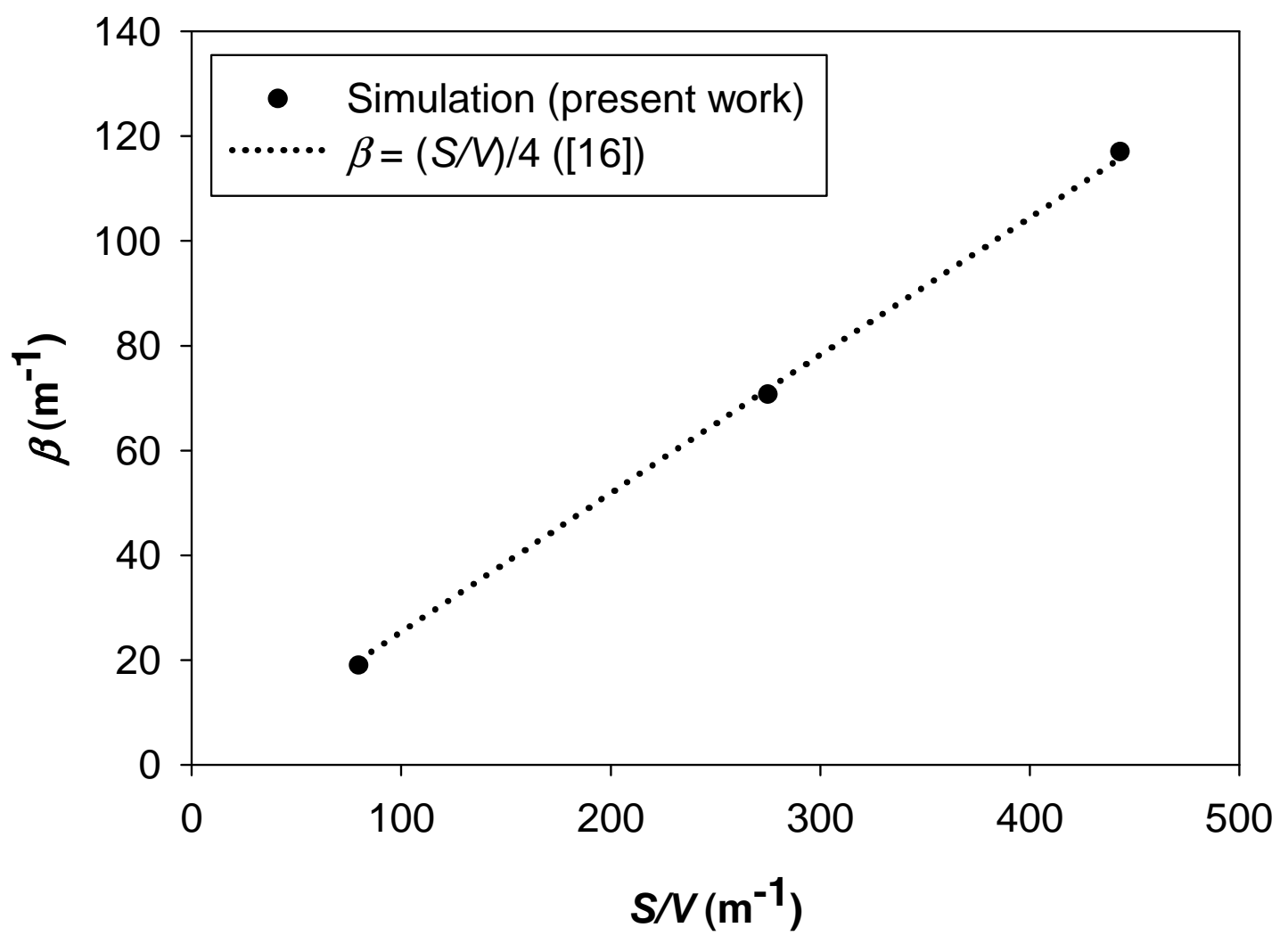

Figure 9 


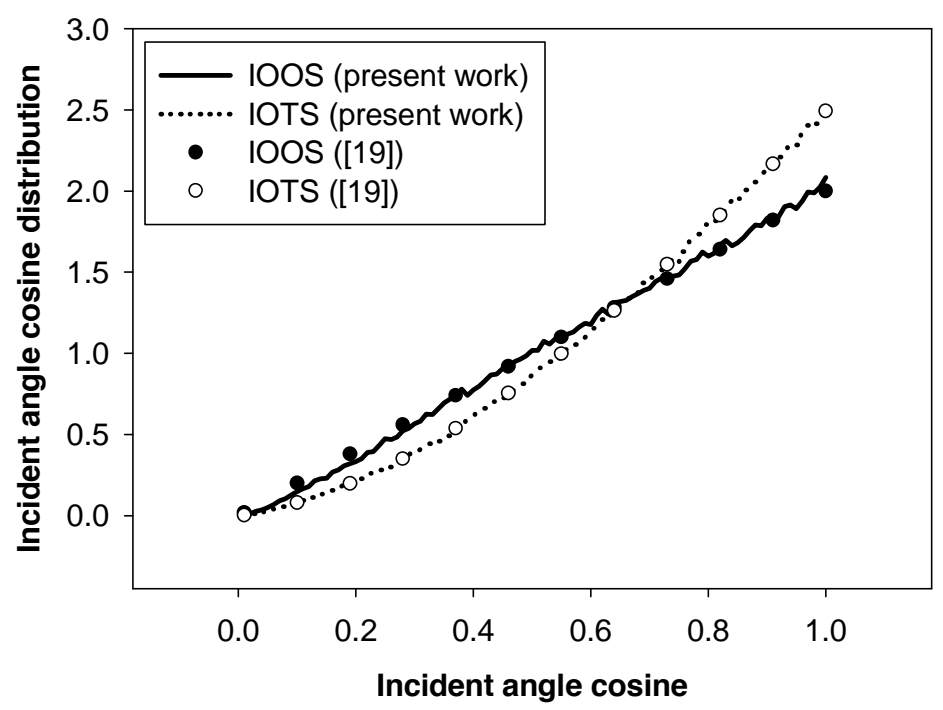

Figure 10 

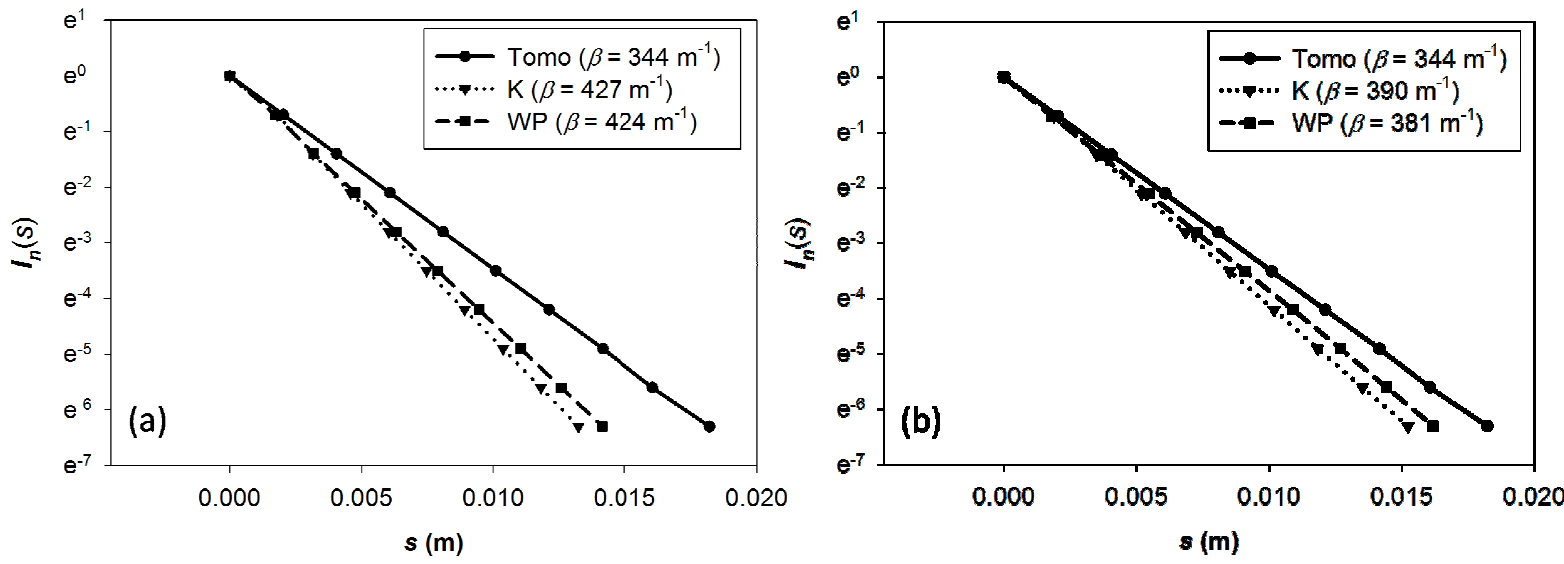

Figure 11 

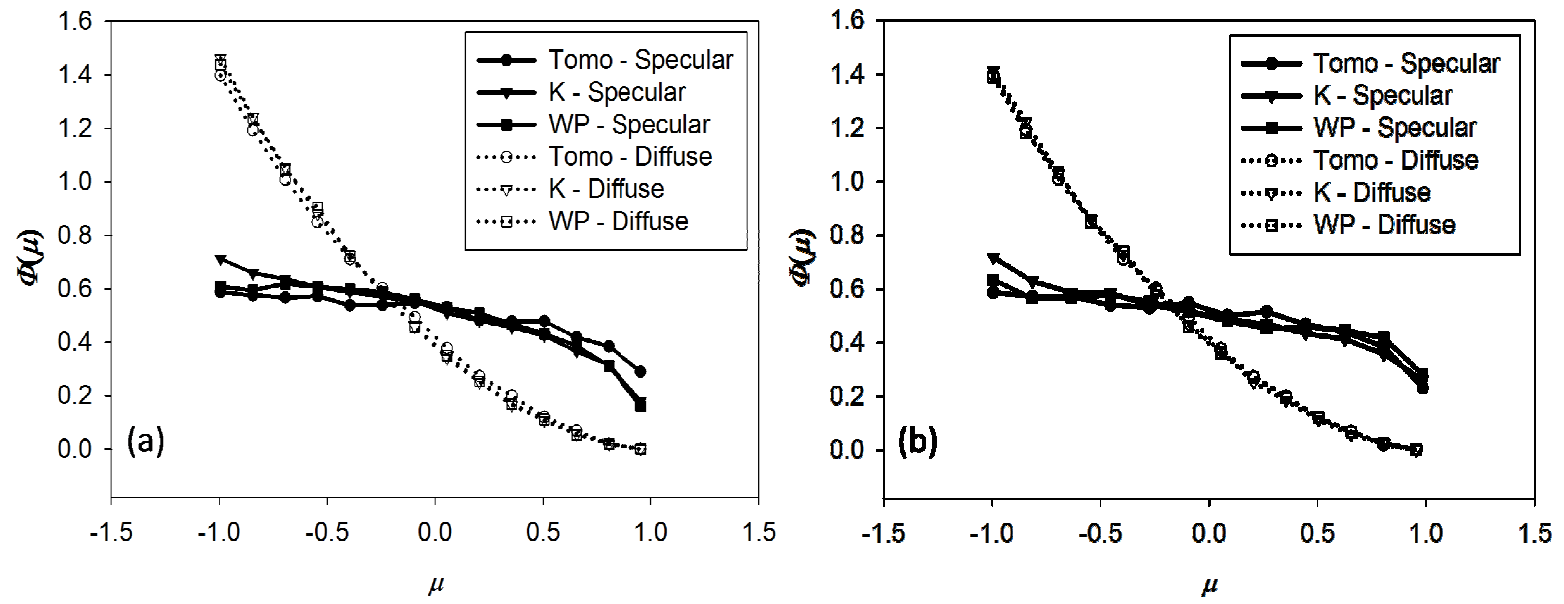

Figure 12 